\title{
Lateral condyle fracture with concomitant postero- medial elbow dislocation in a child: a case report
}

\author{
Ganesh Singh Dharmshaktu* \\ Department of Orthopaedics, Government Medical College, Haldwani, Uttarakhand, India
}

Received: 27 October 2015

Accepted: 25 November 2015

Published online: 29 January 2016

*Corresponding author: Ganesh Singh Dharmshaktu, Department of Orthopaedics, Government Medical College, Haldwani, Uttarakhand, India. Email:drganeshortho@gmail.com

Competing interests: None.

Funding information: None.

Citation: Dharmshaktu GS. Lateral condyle fracture with concomitant postero-medial elbow dislocation in a child: a case report. Journal of Emergency Practice and Trauma 2017; 3(2): 64-65. doi: 10.15171/jept.2015.21.

\begin{abstract}
Simultaneous combination of fracture of the lateral condyle and postero-medial elbow dislocation is a rare event and limited to few reports or case series in the literature. Rarity of the injury also necessitates judicious diagnosis and appropriate management to ensure optimal functional outcome. Various authors have reported about the condition including the management which mostly includes surgical intervention. A report of one such injury pattern in an 8 years old male child with operative management and satisfactory outcome is presented to highlight the presence of this rare combination and adherence to standard treatment protocols to address them. The report is an addition to the limited resources available on similar fractures and highlights the importance of ruling out possible associated injuries in cases of elbow dislocations. A good fixation of lateral condyle fracture is instrumental to avoid late complications leading to poor functional outcome. The early and supervised rehabilitation also plays a role in ensuring successful return to activities of daily living. Keywords: Dislocations, Elbow, Fracture, Lateral condyle, Pediatrics
\end{abstract}

\section{Introduction}

Elbow dislocation constitutes 3\%-6\% of all elbow injures (1). Recent study has indicated an associated injury with elbow dislocation in $75 \%$ of cases (2). Complex elbow dislocation pattern like postero-medial dislocation of elbow with lateral condyle fracture has been a rare injury. Previous reports are limited to isolated case reports or series $(3,4)$. Similar pattern of dislocation has also been reported, but with intraarticular entrapment of lateral epicondyle in one case (5). As the elbow joint is a hinge requiring early management and movement in order to avoid notorious stiffness, prompt diagnosis and emergent reduction of the dislocation followed by good fixation of lateral condyle fragment is necessary for achieving the objective.

\section{Case Presentation}

An 8 years old male child was presented to us by his parents with a history of injury to his left elbow due to falling from a cycle to the ground on outstretched hand. There was swelling, deformity and difficulty in using the extremity since then. There was no history of other related or remote trauma and distal neurovascular status was intact. The radiographs of the affected region were advised and showed a postero-medial elbow dislocation with a fracture of lateral condyle (Figure 1). An emergent reduction of the dislocation was planned with simultaneous fixation after informed consent of the parents and under appropriate anesthesia. Elbow was reduced with gentle traction and flexion with pressure from medial aspect of elbow to guide it into reduction. Clinical assessment of reduction was done with the regain of normal contour of elbow and smooth motion arc. The adequacy and concentricity of the reduction was confirmed by image intensifier. A lateral approach was used to access fractured lateral condyle and that was reduced and held with reduction forceps while fixation with smooth $\mathrm{K}$-wires ( 2 in number) was done under image intensifier guidance. A plaster of Paris back slab was applied as protection splint after meticulous lavage and suturing of the incision. Patient was taken outside the theatre after checking the adequacy of fixation assessed once more.

\section{Results}

The wound healing was uneventful and post operative radiographs were satisfactory for joint congruity and fixation of fracture (Figure 2A). The radiological union was evident on follow up period and the plaster and wires were removed after 4 weeks and supervised physiotherapy was initiated for the gradual regain of function and mobility. The fracture united well and functions of the elbow returned to pre-injury level (Figure 2B). There was no complication related to trauma or surgical technique noted in the follow up of one year. 


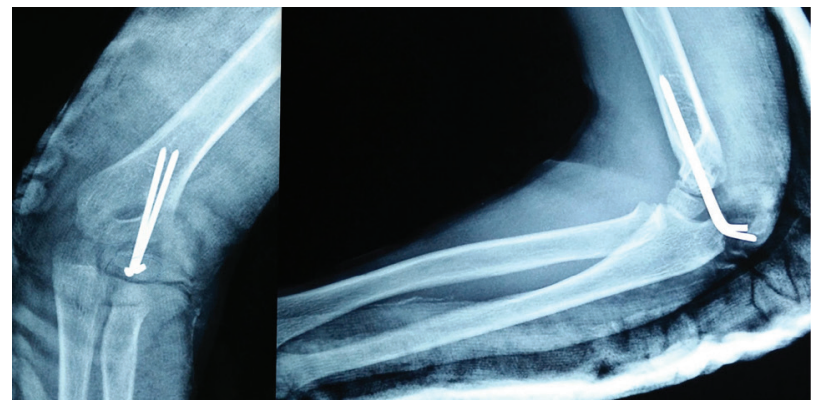

Figure 1. Radiography of elbow AP and lateral views showing postero-medial dislocation with fracture lateral condyle.

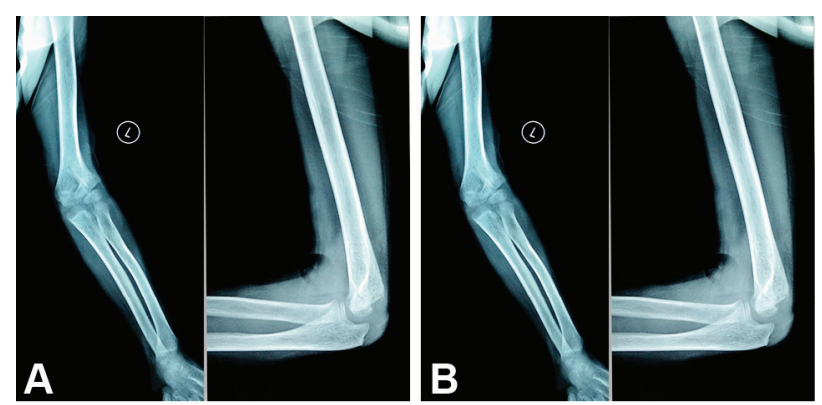

Figure 2. (A) Postoperative radiographs showing the reduction of a fixation. (B) The follow up radiographs showing united fracture and stable joint.

\section{Discussion}

Associated fracture of lateral condyle can be classified as per Milch classification (6) into type 1 and type 2 based on fracture pattern and according to displacement in separate classification (7). Fixation of displaced fractures is standard protocol to avoid late complications notably the nonunion, osteonecrosis of trochlea and angular deformities (8). We planned for emergent reduction of the dislocation and fixation of lateral condyle as it was displaced, followed by compliant physiotherapy after the union has been ensured clinico-radiologically. Fixation of small fragment helps restore integrity of important lateral ligament complex and thus is advisable (9). The similar method of treatment is applied by workers like Kirkos et al (3) and Cheng et al (10) in their series of four and three cases respectively. Other workers witnessed instability of reduction till the fixation of fractures was undertaken in $(11,12)$. One unique observation was noted by Cheng et al (13) in two cases where fractured lateral condyle adhered to radial head through intact capsule and lateral collateral ligaments. Latest reports from India described Milch type 2 fracture with postero-medial dislocation elbow addressed with screws and K-wires (14) in one report and a series of three cases fixed with $\mathrm{K}$-wires respectively (15), both leading to satisfactory results.

\section{Conclusion}

The knowledge of probable associated injuries helps in anticipation and detection leading to optimal management. A good concentric reduction of dislocation and good fixation of lateral condyle fracture is mainstay of the treatment of these injuries in isolation or in combination.

Ethical issues

Informed consent taken for surgery and future publication.

\section{Author's contribution}

GSD is the single author of the manuscript.

\section{References}

1. Wilkins KE. Fractures and dislocations of the elbow region. In: Rockwood CA Jr, Wilkins KE, King RE, eds. Fractures in Children. 7th ed. Philadelphia: Lippincott-Raven; 1996. p. 680 .

2. Rasool MN. Dislocations of the elbow in children. J Bone Joint Surg 2004; 86(7): 1050-58. doi: 10.1302/0301-620x.86b7.14505.

3. Kirkos JM, Beslikas TA, Papavasiliou VA. Posteromedial dislocation of elbow with lateral condyle fracture in children. Clin Orthop Relat Res 2003; (408): 232-6.

4. Hardacre JA, Nahigian SH, Froimson AI, Brown JE. Fractures of the lateral condyle of the humerus in children. J Bone Joint Surg Am 1971; 53(6): 1083-95.

5. Pouliart N, DeBoeck H. Posteromedial dislocation of elbow with associated intraarticular entrapment of lateral epicondyle. J Orthop Trauma 2002; 16(1):53-56.

6. Milch H. Fractures and fracture dislocations of the humeral condyles. J Trauma 1964; 4(5): 592-607. doi: 10.1097/00005373-196409000-00004.

7. Rutherford A. Fractures of the lateral humeral condyle in children. J Bone Joint Surg Am 1985; 67(6): 851-6.

8. Wilkins KE. Fractures and dislocations of the elbow region. In: Rockwood CA, Wilkins KE, King RE, eds. Fractures in Children. 3rd ed. Philadelphia: Lippincott; 1991. p. 618-54.

9. Sharma H, Ayer R, Taylor GR. Complex pediatric elbow injury: an uncommon case. BMC Musculoskelet Disord 2005; 6: 13. doi: 10.1186/1471-2474-6-13.

10. Cheng PG, Chang WN, Wang MN. Posteromedial dislocation of the elbow with lateral condyle fracture in children. J Chin Med Assoc 2009; 72(2): 103-7. doi: 10.1016/S1726-4901(09)70033-4.

11. Rovinsky D, Ferguson C, Younis A, Otsuka NY. Pediatric elbow dislocation associated with a milch type I lateral condyle fracture of the humerus. J Orthop Trauma 1999; 13(6): 458-60. doi: 10.1097/00005131-199908000-00012.

12. Murnaghan JM, Thompson NS, Taylor TC, Cosgrove AP, Ballard J. Fracture lateral epicondyle with associated elbow dislocation. Int J Clin Pract 2002; 56(6): 475-7.

13. Cheng PG, Chen CC, Wu SK, Wang MN. Posteromedial dislocation of the elbow with lateral condyle fracture: The fractured lateral condyle adheres to the radial head. Formosan Journal of Musculoskeletal Disorders 2012; 3(2): 66-9. doi: 10.1016/j.fjmd.2012.03.006.

14. Naik M, Madi SS, Vijayan S, Rao S. A rare pediatric trauma - Lateral condyle humerus fracture with concomitant postero-medial elbow dislocation. Hand Microsurg 2015; 4(3): 75-8. doi: 10.5455/handmicrosurg. 185151.

15. Agrawal PS, Chaudhary SD, Mitra SR. Posteromedial dislocation of the elbow with associated fracture of the lateral humeral condyle in children. MedPulseInternational Medical Journal 2015; 2(7): 388-90. 